\title{
1 Object manifold geometry across the mouse cortical visual \\ 2 hierarchy
}

3 Emmanouil Froudarakis ${ }^{1,4,5 *}$, Uri Cohen ${ }^{2}$, Maria Diamantaki ${ }^{1}$, Edgar Y. Walker ${ }^{4-6}$, Jacob Reimer ${ }^{4,5}$, Philipp

4 Berens $^{5-7}$, Haim Sompolinsky ${ }^{2,3}$, Andreas S. Tolias ${ }^{4,5,8^{*}}$

$5{ }^{1}$ Institute of Molecular Biology and Biotechnology, Foundation for Research and Technology Hellas,

6 Heraklion, Greece

7 ²Edmond and Lily Safra Center for Brain Sciences, Hebrew University of Jerusalem, Israel

$8{ }^{3}$ Center for Brain Science, Harvard University, Cambridge, MA, USA

$9{ }^{4}$ Department of Neuroscience, Baylor College of Medicine, Houston, TX, USA

$10{ }^{5}$ Center for Neuroscience and Artificial Intelligence, Baylor College of Medicine, Houston, TX,USA

$11{ }^{6}$ Institute for Ophthalmic Research, University of Tübingen, Germany

$12{ }^{7}$ Department of Computer Science, University of Tübingen, Germany

$13{ }^{8}$ Department of Electrical and Computer Engineering, Rice University, Houston, TX, USA

14 *To whom correspondence should be addressed; E-mail: frouman@imbb.forth.gr, astolias@bcm.edu

\section{Abstract}

16 Despite variations in appearance we robustly recognize objects. Neuronal populations responding to objects

17 presented under varying conditions form object manifolds and hierarchically organized visual areas are

18 thought to untangle pixel intensities into linearly decodable object representations. However, the associated

19 changes in the geometry of object manifolds along the cortex remain unknown. Using home cage training we

20 showed that mice are capable of invariant object recognition. We simultaneously recorded the responses of

21 thousands of neurons to measure the information about object identity available across the visual cortex and

22 found that lateral visual areas LM, LI and AL carry more linearly decodable object identity information

23 compared to other visual areas. We applied the theory of linear separability of manifolds, and found that the

24 increase in classification capacity is associated with a decrease in the dimension and radius of the object

25 manifold, identifying features of the population code that enable invariant object coding. 


\section{Introduction}

Object recognition is an ethologically-relevant task for many animals. This is a challenging problem

28 because an individual object can elicit myriads of images on the retina due to so-called nuisance

29 transformations such as changes in viewing distance, projection, occlusion and illumination. The collection of

30 neural responses associated with a single object is known as the object manifold. A prevailing hypothesis is

31 that along the visual hierarchy, object manifolds are gradually untangled to produce increasingly invariant

32 object representations, which are linearly decodable (DiCarlo and Cox, 2007). This hypothesis is primarily

33 based on work in non-human primates, which is a powerful model to study object recognition especially given

34 the similarities in visual perception among primates. These studies have revealed that the selectivity for object

35 identity increases as visual signals are conveyed from primary visual cortex (V1) to inferotemporal cortex

36 (Hung et al., 2005; Rust and DiCarlo, 2010). Despite this significant progress, the underlying changes in the

37 geometry of the object manifolds along the visual cortical hierarchy that leads to object recognition and a

38 circuit-level mechanistic understanding of how they are generated remain largely unknown. The mouse animal

39 model is ideally suited to dissect circuit mechanisms due to its genetic tractability and the numerous methods

40 available to perform large scale recordings, manipulations and anatomical tracing with cell-type precision

41 (Fenno et al., 2015; Sofroniew et al., 2016). Therefore developing visually guided behaviors in rodents is

42 important (Zoccolan et al., 2009) and identifying the relevant network of visual areas involved in object

43 recognition analogous to the ventral stream of primates is critical. In this direction, we developed an

44 automatic high-throughput training paradigm and demonstrated that mice can be trained to perform a

45 two-alternative forced choice (2AFC) object classification task, which is typically used in primates to test

46 object identification. While visually-guided operant behavioral tasks have been used previously in mice (Hu et

47 al., 2018; Leger et al., 2013), here we show that mice can also learn to correctly discriminate objects under a

48 2AFC paradigm. Critically, this capability persisted even when they were presented with previously-unseen

49 transformation of objects.

We simultaneously recorded the activity of thousands of neurons across all cortical visual areas of the

51 mouse: primary (V1), anterolateral (AL), rostrolateral (RL), lateromedial (LM), lateral intermediate (LI),

52 posteromedial (PM), anteromedial (AM), posterior (P), postrhinal (POR) and laterolateral anterior (LLA)

53 visual areas, while presenting images of moving objects undergoing numerous identity-preserving

54 transformations such as rotation, scale and translation across different illumination conditions. By decoding

55 the identity of the objects from the recorded neural activity using a linear classifier we found that the lateral

56 extrastriate visual areas (LM, AL, LI) carried more linearly decodable information about objects identity

57 compared to V1 and all other higher order areas we studied, consistent with results in rats (Tafazoli et al.,

58 2017). We used the recently developed theory of linear separability of manifolds and found that in areas LM

59 and AL the increase in classification capacity is associated with improved manifold geometry, where both the

60 manifold radii and dimensions are reduced compared to other visual areas. Additionally, by recording

61 simultaneously from many visual areas, we found that the population dynamics differed across the visual 
62 hierarchy, where information about object identity accumulated faster in areas which are more object selective

63 compared to V1.

\section{Results}

65 We generated rendered movies of 3D objects by varying their location, scale, 3D pose and illumination in a 66 continuous manner across time (Fig. 1a, Supp. Movie 1). We developed a 2AFC automatic home cage

67 training system in which water restricted mice had to lick a left or a right port depending on the object that 68 was shown on a small monitor in front of their cage (Fig. 1b). Upon a correct choice animals immediately 69 received a small amount of water reward. Naive animals initially licked the left and right probes at random, 70 but within two weeks of training animals learned to preferentially lick the correct port matched to object 71 identity (Fig. 1c) and trained animals maintained consistent performance on the task across days (Fig. 1d).

72 An important property of object recognition is the ability to generalize across views of objects that have never 73 been seen before. After the animals learned to discriminate objects from the movie clips - which contained a 74 specific set of object transformations, new movie clips with unique parameters across translation, scale, pose 75 and illumination, were presented to the animals. We could not detect any differences in performance between 76 the previously seen object transformations (Fig.1e, familiar transformations) and novel object transformations 77 (Fig.1e, novel transformations). This ability to generalize across identity-preserving transformations indicated 78 that mice learned an internal object-based model and did not rely simply on low-level features of the rendered 79 movies they observed during training.

If mice were capable of discriminating between objects, there should exist a set of areas along their

81 visual processing hierarchy that can extract this information. It has been suggested that one way of extracting

82 the object information irrespective of its transformations is to have neural representations for each object that

83 are untangled, i.e. can be read-out using a linear decoder (DiCarlo and Cox, 2007). To test this idea, we used

84 transgenic mice expressing GCamp6 in pyramidal neurons and recorded the activity from hundreds of neurons

85 in each visual areas separately or from thousands of neurons across the whole visual cortical hierarchy of the

86 mouse using a large field of view microscope (Sofroniew et al., 2016, Fig. 1f, g), while the animals passively

87 viewed the moving objects (Fig. 1a). We identified the borders between visual areas using wide-field

88 retinotopic mapping as previously described (Fahey et al., 2019; Garrett et al., 2014; Wang and Burkhalter,

89 2007) (Fig. 1f). Neurons in all of the identified visual areas showed significantly more reliable responses

90 when compared to neurons that were not assigned to any visual area, based on the area segmentation (Supp.

91 Fig. 1a). 
a

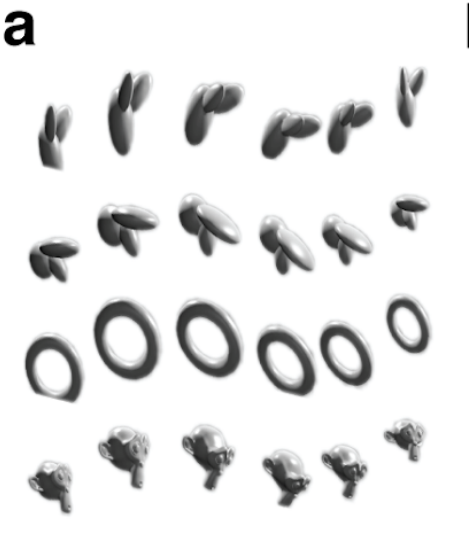

b
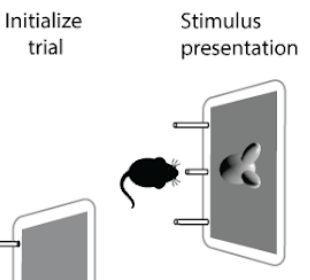

ion
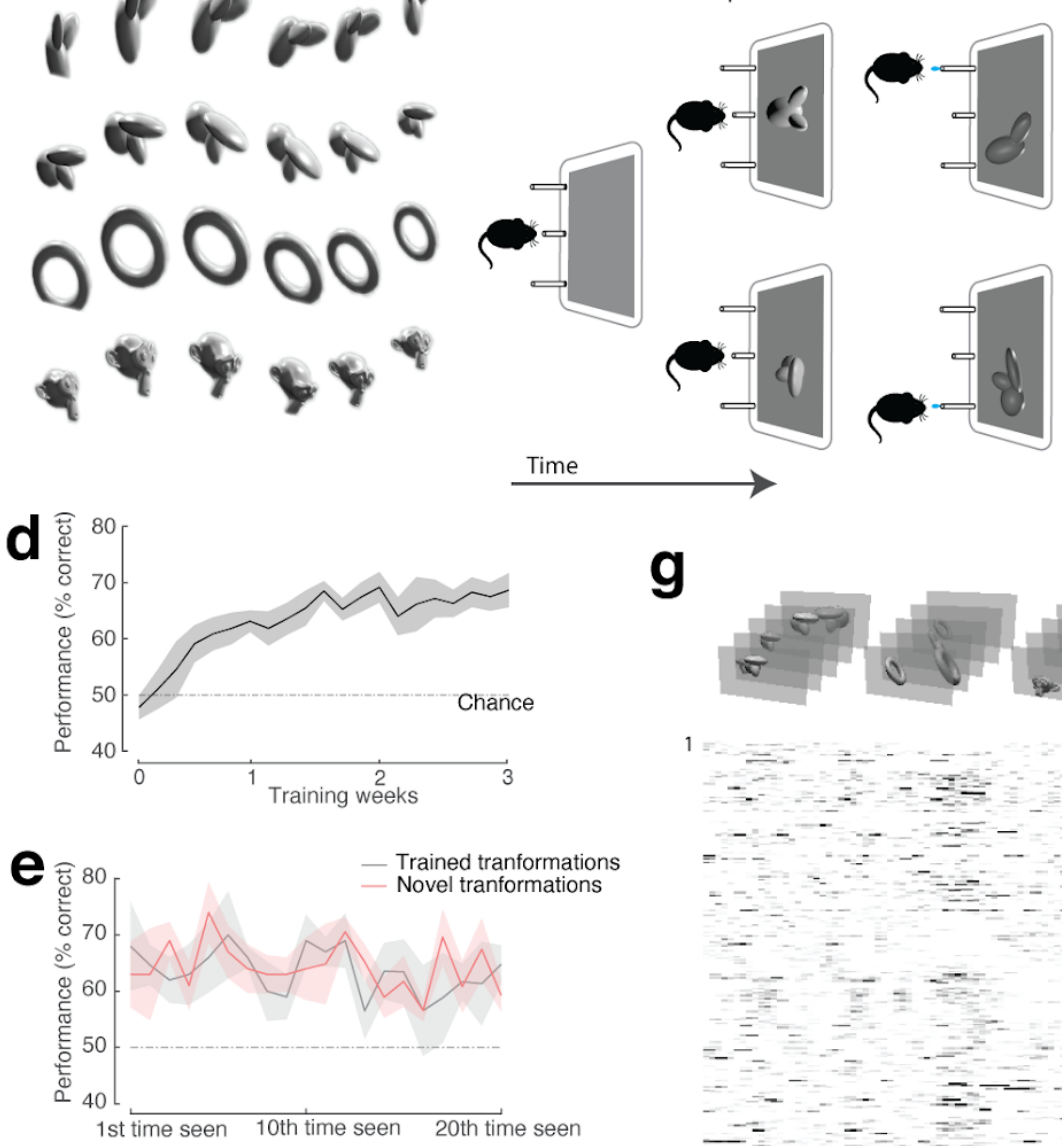

f

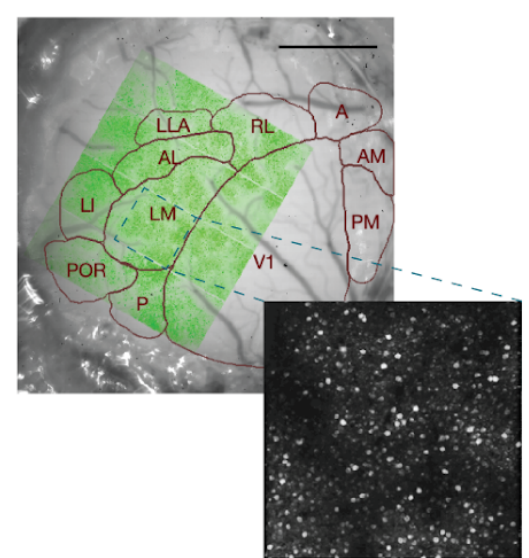

\section{g}

C $1^{a t}$ week of traning
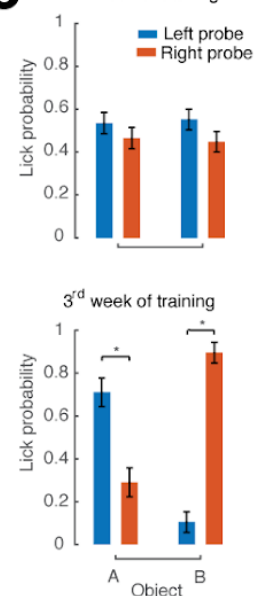
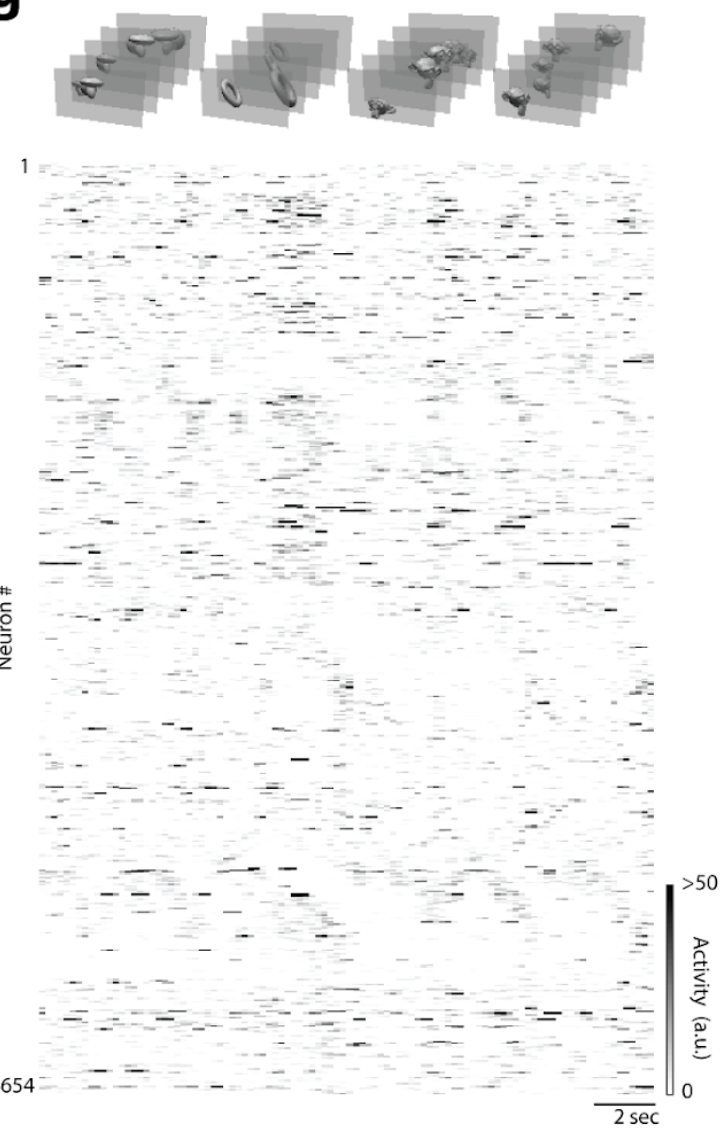

92 Figure 1: Experimental procedure for behavioral training and two-photon imaging. (a) Single frames from movies with the

93 objects that were presented to the animals. (b) Behavioral training sequence. (c) Probability of licking either probe during the early 94 training period (upper barplot) and later training period (lower barplot) for 1 animal. Error bars represent S.E.M. Student t-test * p < 950.05 (d) Performance as a function of training time, $\mathrm{N}=8$ animals. (e) Performance across repetitions of previously seen (gray) and 96 previously unseen (red) object trajectories during one session. $\mathrm{N}=6$ animals. For both (d) and (e) shaded areas represent S.E.M. (f) 97 Example large field of view recording (green) with area boundaries overlaid. Scale bar represents $1 \mathrm{~mm}$. A small inset depicts the 98 two-photon average image for a small segment of the large field of view captured with the mesoscope. (g) Example responses of all 99 neurons to moving objects (shown on top) from the recording shown in (f). Each clip is presented for 3-5 seconds before a short pause 100 switches to a new clip that might be the same or a different object identity. 
bioRxiv preprint doi: $h t t p s: / / d o i . o r g / 10.1101 / 2020.08 .20 .258798$; this version posted August 21, 2020. The copyright holder for this preprint (which was not certified by peer review) is the author/funder, who has granted bioRxiv a license to display the preprint in perpetuity. It is made available under aCC-BY-NC-ND 4.0 International license.

To measure how linearly discriminable the responses to the different objects were, we used

102 cross-validated logistic regression to classify the object identity from the responses of neurons in each visual

103 area. As expected, discriminability increased as a function of the number of neurons sampled (Fig. 2a), but

104 only the higher visual areas, LM, LI and AL, showed consistently higher discriminability levels compared to

105 V1 responses (Fig. 2a, b, c). In contrast, areas RL, AM, P, POR and LLA had significantly lower

106 discriminability levels when compared to V1 (Fig. 2b). The differences in decoding between these areas

107 persisted at the single neuron as well (Fig. 2d, Supp. Fig. 2a).



C Population (128 neurons)



d

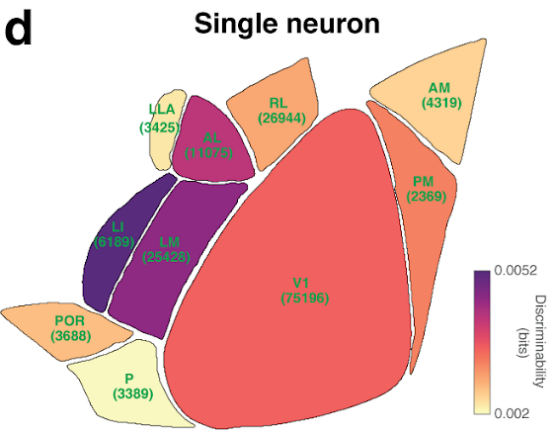

b
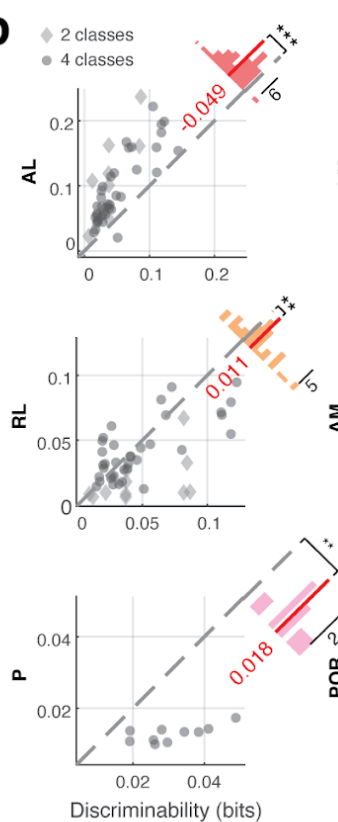

e
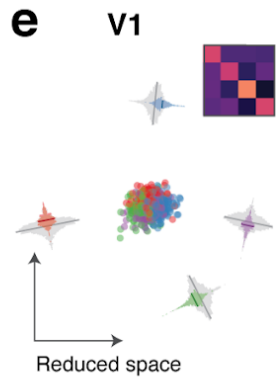
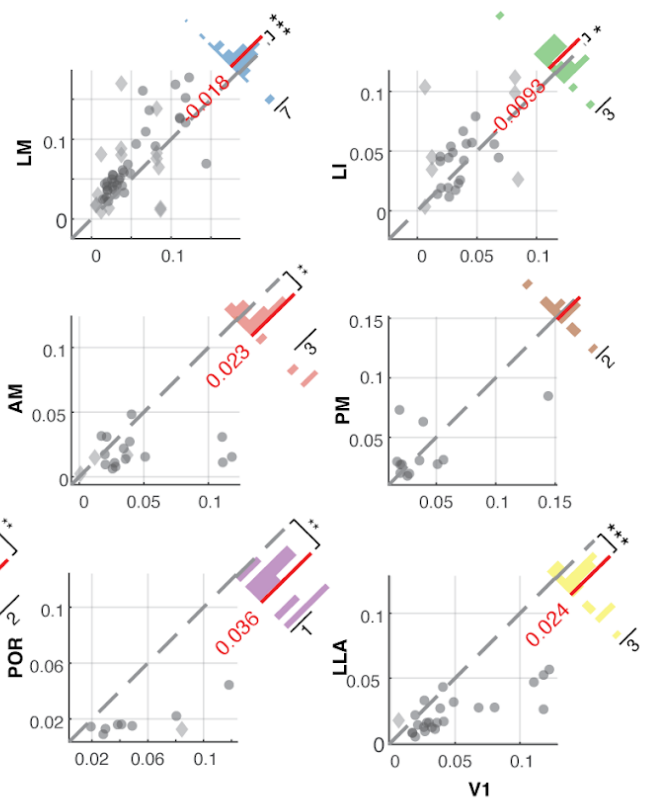

LM

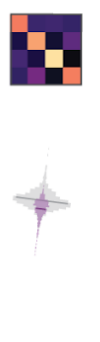

AL
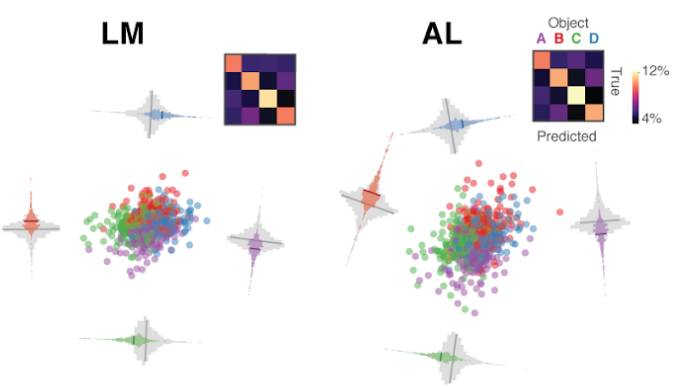

108 Figure 2: Object identity decoding across the visual hierarchy. (a) Discriminability of object identity as a function of the number of

109 neurons sampled. Each line represents the average across all recorded sites. (b) Scatter plot of the discriminability of different areas

110 with a 64 population of neurons compared to V1 for all the recording sites. Insert histogram represents the difference between the

111 discriminability of each area and V1. Red line and number indicate the mean difference. Diamonds represent the results with 2 objects

112 whereas circles represent the results with 4 objects. Outliers have been omitted for better visualization. Wilcoxon signed rank test $* * *$

$113 \mathrm{p}<0.001, * * \mathrm{p}<0.01, * \mathrm{p}<0.05$. (c) Average discriminability of all visual areas with a 128 population of neurons. The number below

114 each area represents the recording sites sampled. (d) Same as in (c) but when using a single neuron at a time to decode the object

115 identity. The number below each area represents the cells sampled. (e) Low-dimensional representation of the 128-dimensional neural

116 activity space, illustrating the separation of the responses to four different objects for three example areas. Each dot represents the

117 average of the activity in one 500msec bin. The side histograms represent the distances of the data projected onto each of the four

118 object category axes for the same-class (colored) and different-class (gray). Each insert represents the confusion matrix after decoding. 
We performed several control analyses: first, our results might be due to differences in the retinotopic coverage across areas. As has been reported before (Garrett et al., 2014), the coverage of the visual field is different between visual areas. To control for this, in some experiments we also mapped the receptive fields

122 (RFs) of all recorded neurons using a dot stimulus (see methods) and repeated our decoding analysis using 123 only neurons from each area with RF centers within the same 20 degree area of visual space. When we 124 restricted our analysis in this way, areas LM, LI and AL still showed significantly higher discriminability 125 (Supp. Fig. 2b). Another potential confound might be differences in receptive field sizes across areas (Murgas 126 et al., 2020; Wang and Burkhalter, 2007). An area with larger receptive fields might be better at representing

127 objects simply because more neurons are responding to the object at any moment. Indeed, when we examined 128 decoding performance conditioned on the object size, we observed an increase in discriminability for all 129 visual areas as a function of object size (Supp. Fig. 3a), in agreement with the increased performance we 130 found when sampling from more neurons (Fig. 2a). However, if changes in receptive field size alone result in 131 an increase in object discriminability we would expect that area PM that has very large receptive fields 132 (Murgas et al., 2020) would also have high object discriminability which was not the case in our data (Fig. 2).

133 To further investigate the influence of receptive field size on discriminability, we modeled the effect of 134 changing receptive field (RF) size in a simulated population of neurons with filters learned by a sparse coding 135 model. Increasing the size of the receptive fields by either scaling or pairwise linearly combining them (Supp. 136 Fig. 4a, see Methods) led to either a decrease in discriminability or had no significant effect respectively 137 (Supp. Fig. 4b). These results argue that our in vivo results cannot be trivially explained by differences in the 138 receptive field sizes across visual areas. Additionally, higher visual areas have been reported to have different 139 temporal frequency selectivities (Andermann et al., 2011; Han et al., 2018; Marshel et al., 2011). To determine 140 whether the range of speeds that objects were moving in the movies that we showed influenced our results, we 141 computed the decoding performance for each area as a function of the object speed, but did not find any 142 significant differences (Supp. Fig. 3b). Therefore, we interpret the increase in discriminability in AL, LI, and 143 LM indicating that these visual areas are specialized for the processing of visual object information with 144 neural representations that are easier to decode (Fig. 2e). 
a

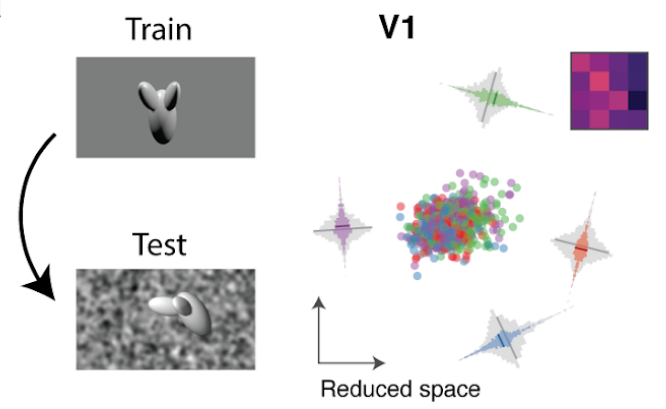

LM

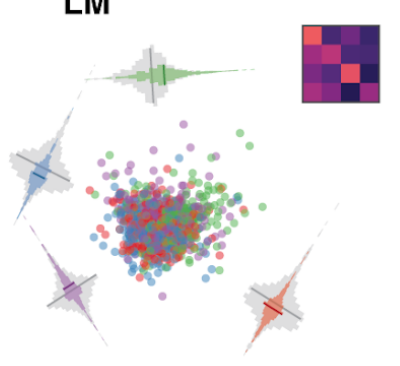

d

b

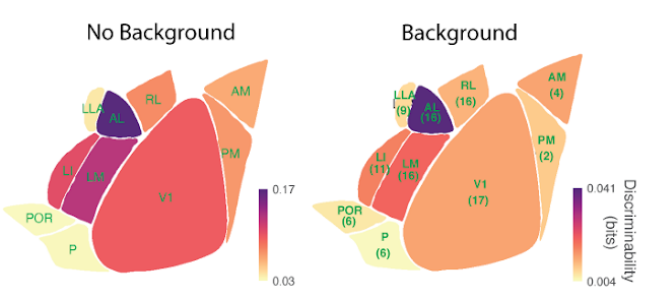

C

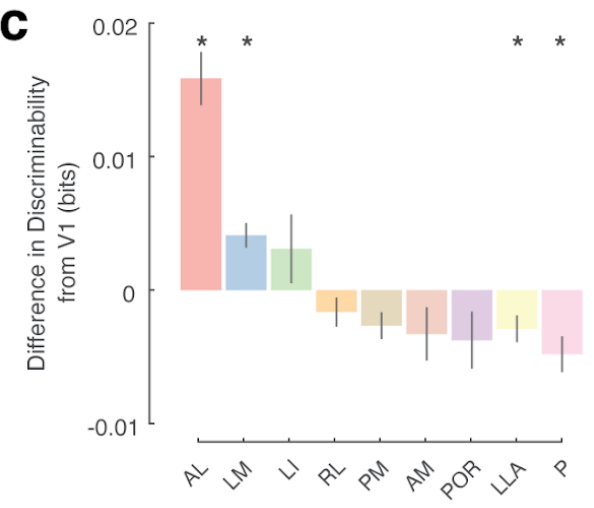

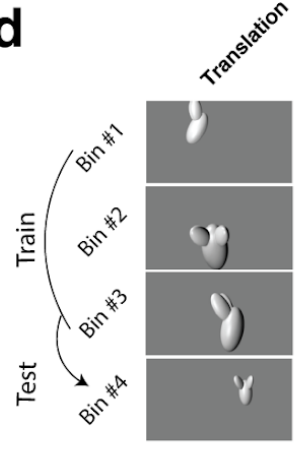

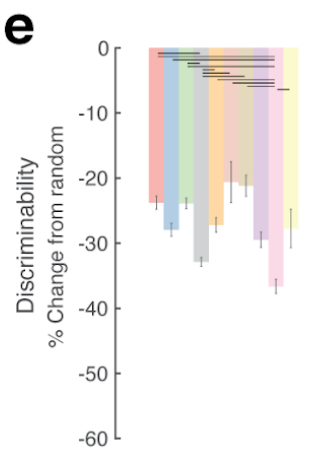

AL
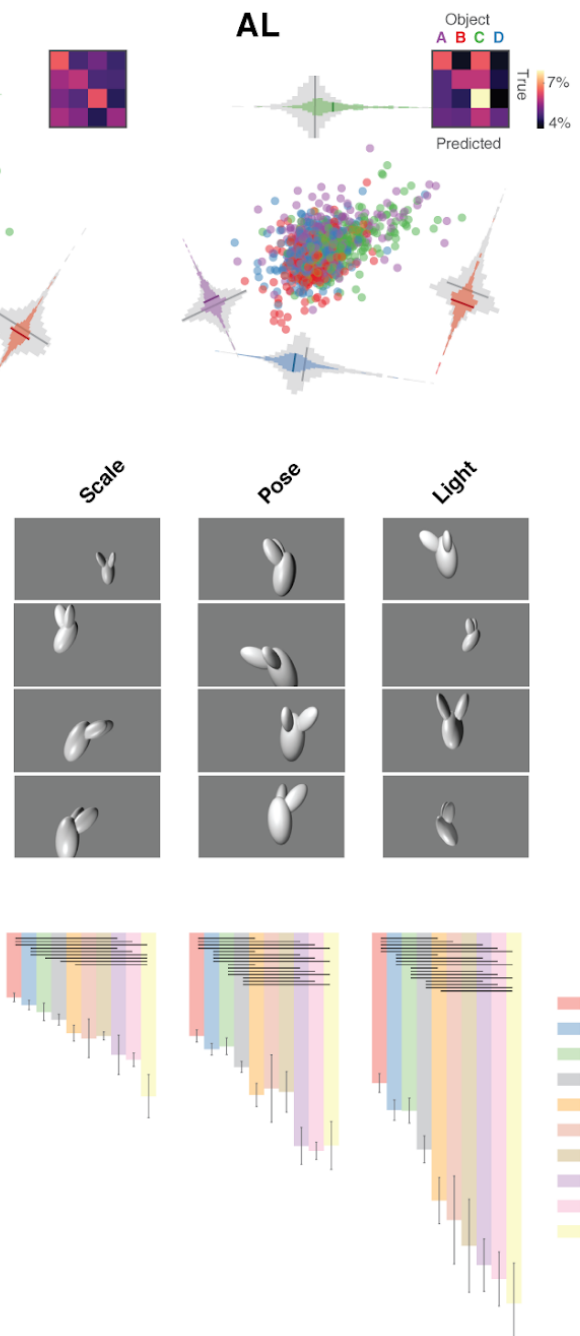

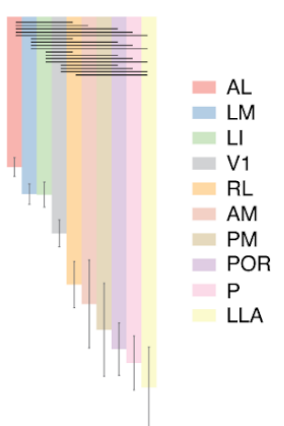

145 Figure 3: Generalization performance across background noise and identity-preserving transformations. (a) Generalization test

146 across background noise. The decoder was trained on the responses to objects without background and tested on the responses to

147 objects that contained background noise. Low-dimensional representation of the responses to the object w/ background are shown on

148 the right similar to Figure 2e. Each insert represents the confusion matrix after decoding. (b) Average discriminability of all visual

149 areas for objects w/o and w/ background, on the same recorded sites. (c) Barplot indicating the difference in discriminability between

150 all visual areas and V1 on the responses to objects w/ background. Kruskal-Wallis with multiple comparisons test *p < 0.001. (d)

151 Example parameter space of the four nuisance classes: Translation (x/y), Scale, Pose (tilt/rotation) and Light (four light sources). The

152 decoder was tested on a parameter space of each of the four nuisance variables that had not been part of the training set. (e) Bar plot

153 indicating the performance when testing on untrained parameter space, compared to the performance of the random sampling across all

154 classes. Lines indicate $\mathrm{p}<0.05$ Kruskal-Wallis with multiple comparisons test.

An important property of visual areas that extract information about object identity is robustness to

156 background clutter in the visual scene. To assess the effect of background-clutter, we first trained a logistic

157 regression decoder on responses to objects with a gray background as we used previously. We then evaluated

158 the performance of the decoder on the responses to movies in which we embedded the objects on top of

159 background clutter (Fig. 3a, Supp. Movie 2). While the discriminability decreased for all visual areas when

160 compared to noise-free stimuli (Fig. 3b), areas LM and AL maintained significantly higher discriminability 
161 compared to V1 and all other visual areas (Fig. 3a,b,c), indicating that in addition to being highly invariant to

162 changes in the appearance of the object, the object representation in these areas is also more robust than in V1

163 and other visual areas to clutter. We also studied the relationship between discriminability and reliability of the

164 neural responses. Although the decoding performance of the objects without the background correlated well

165 with the reliability of the responses for both V1 and the lateral visual areas, when background noise was

166 introduced this relationship broke down for V1 but not for the lateral visual areas Supp. Fig. 1b).

Neurons in an area that is specialized for representing objects should depend less on object parameters

168 that preserve the identity such as position, scale, pose and illumination conditions (DiCarlo and Cox, 2007;

169 Hénaff et al., 2019; Rust and DiCarlo, 2010; Tafazoli et al., 2017). As a result, an object decoder built on a

170 subset of the nuisance parameter space (for example a limited range of translations, sizes, and rotations)

171 should generalize across nuisance parameters. To test this we split the data into four non-overlapping bins for

172 each of the nine continuously-varying parameters that defined the object stimulus (for example for the size

173 object parameter, very small, small, medium, and large objects; Fig. 3d), while the remaining parameters were

174 randomly sampled. For each parameter, we then used data from three of the bins to train the decoder, and

175 tested the prediction performance on the held-out bin of the data. We compared this performance to a baseline

176 discriminability using a 4-fold cross validation, when the values for each parameter were randomized before

177 binning so that the training and test set both spanned the same parameter range. Comparing the out-of-training

178 set performance to this baseline allowed us to assess the ability of the decoder to generalize, and thus assess

179 the invariance of the representation in each area (Supp. Fig. 5, Fig. 3e; negative values). Areas AL, LM and

180 LI consistently showed the best generalization performance (smallest reduction in performance for

181 out-of-training-set condition vs baseline), when changing the scale, pose, light but not translation (Fig. 3e).

182 The larger receptive field sizes of areas PM and AM (Murgas et al., 2020; Wang and Burkhalter, 2007) might

183 contribute to the improved translation invariance that we observed relative to the other parameters.

Chung and colleagues (Chung et al.,2018) recently developed the theory of linear separability of

185 manifolds and defined a measure called the classification capacity which quantifies how well a neural

186 population supports object classification. The classification capacity measures the ratio between the number

187 of objects and the size of the neuronal population that is required for reliable binary classification of the

188 objects, and is tightly related to the geometry of a neuronal population responding to an object presented

189 under varying conditions (object manifold). In deep neural networks trained on object classification tasks, it

190 has been shown that the classification capacity improves along the network's processing stages (Cohen et al.,

191 2020). Our data, consisting of responses of large neuronal populations in different visual areas to objects

192 under various transformations, are well suited for applying this method to characterize the object manifolds in

193 different visual areas. We used the neuronal responses of 64 simultaneously recorded neurons from each

194 visual area to four objects under the identity-preserving transformations introduced earlier (object position,

195 scale, pose and illumination conditions, with and without background noise). In agreement with our decoding

196 results, we found that the classification capacity increased in higher visual areas AL and LM compared to V1,

197 but decreased in the rest of the areas (Fig. 4a,b). The theory of linear separability of manifolds (Chung et al., 
bioRxiv preprint doi: https://doi.org/10.1101/2020.08.20.258798; this version posted August 21, 2020. The copyright holder for this preprint (which was not certified by peer review) is the author/funder, who has granted bioRxiv a license to display the preprint in perpetuity. It is made available under aCC-BY-NC-ND 4.0 International license.

198 2018) also enabled us to characterize the associated changes in the geometry of the object manifolds to 199 understand how object invariant representations arise along a processing hierarchy (Cohen et al., 2020) (i.e. 200 relate the manifolds' classification ability to the geometry of object manifolds). In particular, classification 201 capacity depends on the overall extent of variability across the encoding dimensions, the radius of the 202 manifold, but also the number of directions in which this variability is spread, the dimension of the manifold. 203 These geometric measures influence the ability to linearly separate the manifolds (Fig. 4c). In our results, we 204 find that the increase in classification capacity can be traced to changes in the manifolds' geometry, both as a 205 decrease of the dimension and radius of object manifolds (Fig. 4d).

a
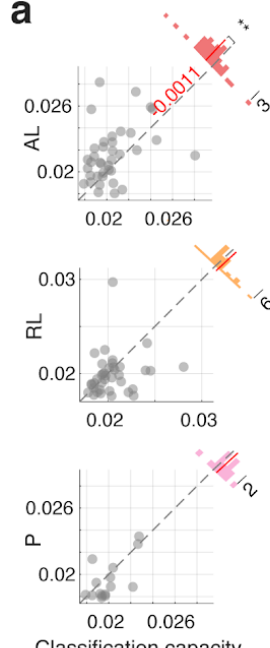
b

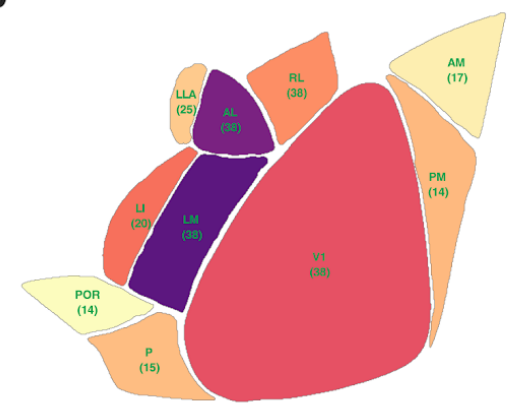

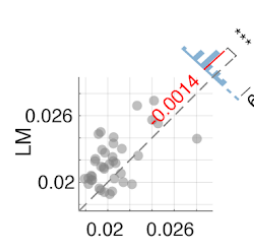
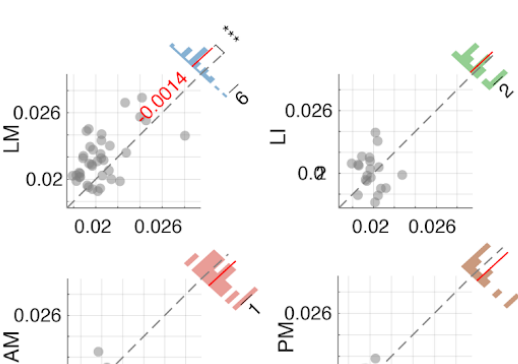

$\sum_{\varangle}$


$\exists .0$



C
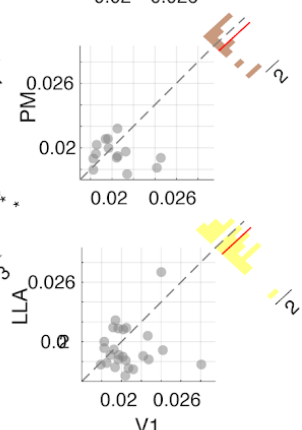

d
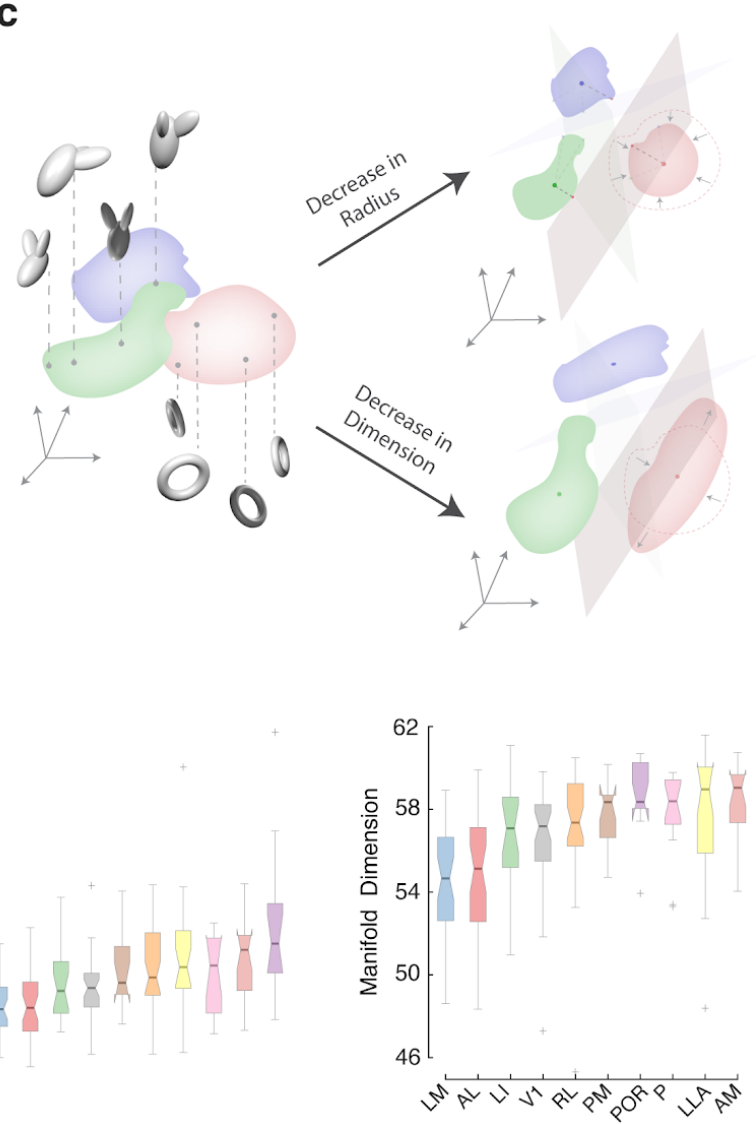

206 Figure 4: Classification capacity and geometry of manifolds across the visual hierarchy. (a) Scatter plot of the classification

207 capacity of different areas compared to V1 for 4 objects. Insert histogram represents the difference between the classification capacity

208 of each area and V1. Red line and number indicate the mean difference. Wilcoxon signed rank test $* * * \mathrm{p}<0.001, * * \mathrm{p}<0.01, * \mathrm{p}<$

209 0.05. (b) Average classification capacity of all visual areas with a 64 population of neurons. The number below each area represents

210 the recording sites sampled. (c) Illustration of low dimensional representations of object manifolds for two visual areas. Left: each

211 point in an object manifold corresponds to neural responses to an object under certain identity-preserving transformations. Right:

212 demonstration of two possible changes in the manifold geometry in a higher order area, reduction of the radius of one manifold

213 through reduction of its extent in all directions (top) and reduction of the dimension of one manifold by concentrating variability at

214 certain elongated axis, reducing the spread along other axes. Such changes have predictable effects on the ability to perform linear

215 classification of those objects. (d) Box plots of the manifold radius (left), and manifold dimension (right) of all areas, sorted in

216 ascending order of the median value. 
One question that arises is how these visual areas are able to form invariant representations that can

218 generalize across background noise or nuisance parameters. One way for these areas to optimize the

219 representations is by taking advantage of the temporal continuity that exists for natural objects by integrating

220 information over time (DiCarlo et al., 2012; Orlov and Zohary, 2018). We analyzed the temporal dynamics of

221 the decoding performance of 50 simultaneously recorded neurons for objects overlaid on background noise.

222 From one trial to the next the nuisance parameters varied continously but the object identity was preserved

223 (cis trials) or switched (trans trials) (Fig. 1g). When we compared the discriminability as a function of time for

224 cis/trans trials, we found that indeed in the trials in which the identity of the object was switched (trans trials),

225 discriminability was overall lower across all visual areas in the early phase of the trials compared to the late

226 phase of the trials, providing evidence for temporal integration during a trial (Supp. Fig. 6). In the late period

227 discriminability in area AL was significantly closer to the discriminability levels of the cis trials than all the

228 rest of the visual areas, suggesting that activity in AL more quickly evolved to more disentangled

229 representations (Supp. Fig. 6b, Early/Late).

A natural question is what are the dependencies between the representations of objects across multiple

231 visual areas. If information about object identity propagates across areas, then we expect to see consistent

232 temporal relationships in the evolution of object discriminability across these areas. We estimated each area's

233 confidence about the identity of the object at each time point, as the distance of the population activity from

234 the decision boundary (Fig. 5a), and we examined the evolution of this metric across time in each area.

235 Specifically, we estimated the distance to the decision boundary at different moments within the trial for the

236 class that was presented. This decision boundary was a linear hyperplane in the 128 dimensional neural

237 activity space (Fig. 5a). We then computed the correlation between the resulting temporal vectors of the score

238 values across all simultaneously recorded visual areas (Fig. 5b, Score Correlation). The highest correlations in

239 this moment-to-moment discriminability score were between AL, LM, RL and V1 (Fig. 5c).

240 Given that activities of neurons across areas can co-fluctuate because of global brain states, these

241 score correlations could just be the result of raw activity correlations across areas. To test this we computed

242 the activity correlations between the responses of pairs of neurons across visual areas. We observed a different

243 correlation pattern that was distinct from the structure of the score correlation (Fig. $\mathbf{5 b}$, Score correlations vs

244 Pairwise activity correlations). Additionally, there are multiple dependencies that can affect the score

245 correlation between the activity of pairs of areas. In order to measure the strength of the linear relationship

246 between each pair of areas after adjusting for relationships with the rest of the areas, we computed the partial

247 score correlations. The correlation pattern remained unchanged with strong dependencies between V1-LM,

248 V1-RL and LM-AL suggesting that these areas work together as a network of areas specialized for object

249 recognition (Fig. 5c). Interestingly, we did not find a strong relationship between V1-AL (Fig. 5c). 


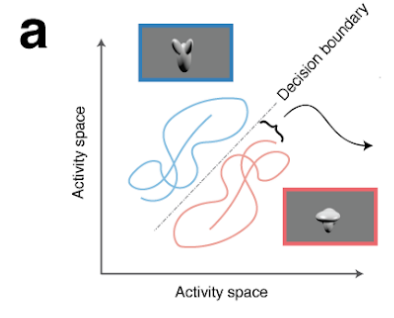

b

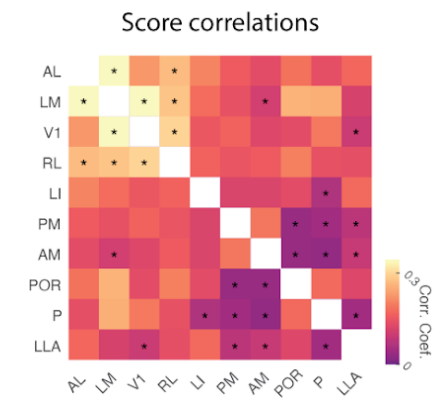

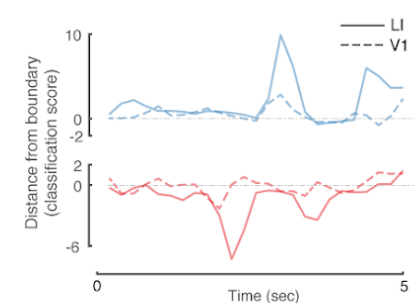

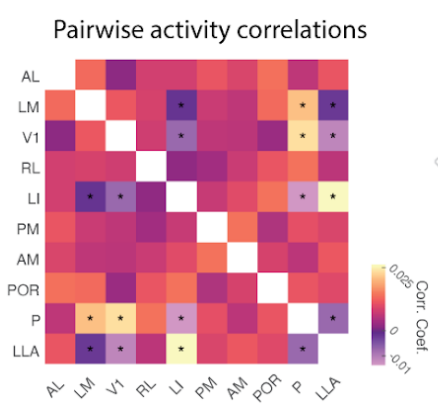

C

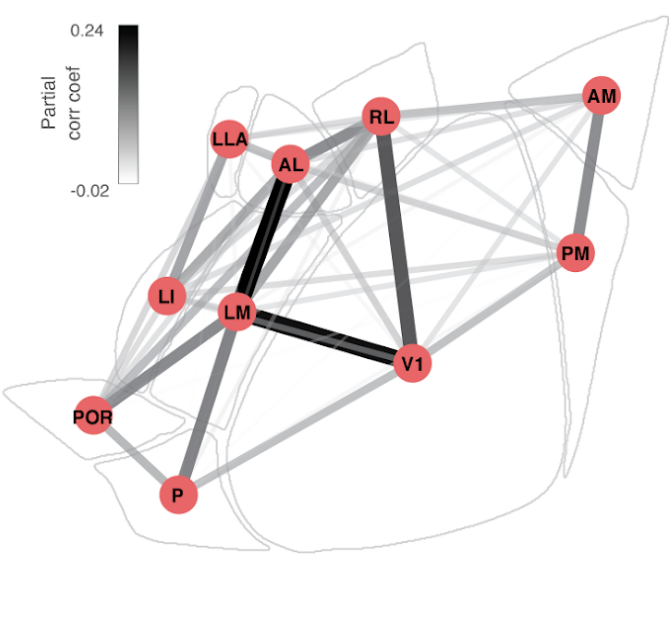

250 Figure 5: Temporal dynamics and cross-area dependencies. (a) Schematic representation of the classification scores as the 251 distances of the response trajectories to the decision boundary (left) and their resulting temporal dependencies across different areas

252 (right). (b) Score correlations across all recorded areas (left) and raw pairwise correlations of the single neuron activity between areas 253 (right). Significance was estimated by bootstrapping across all correlations, ${ }^{*} \mathrm{p}<0.025 / 45$. (c) Schematic representation of the score 254 partial correlation coefficients between areas.

\section{Discussion}

The ability to recognize, discriminate, and track objects across time is evidently a key adaptive trait

257 that is fundamental to identifying food items or conspecifics (Jones and Ratterman, 2009) and the ability to

258 recognize objects has been observed not only in higher mammals such as humans and monkeys, but also

259 rodents, birds, fish and insects (Bevins and Besheer, 2006; Blaser and Heyser, 2015; Newport et al., 2018;

260 Soto and Wasserman, 2014; Werner et al., 2016; Zoccolan et al., 2009). While the implementation of how

261 information is extracted from the visual scene may vary across species, the computational problem remains

262 the same: construct an invariant representation of objects under a wide range of identity-preserving

263 transformations. While there is plenty of evidence that mice can detect novel objects (Leger et al., 2013), and

264 that mice rely on their vision to hunt crickets (Hoy et al., 2016), until our study there was no direct evidence

265 that mice are capable of invariant object recognition.

In this work, we showed that mice can be trained to recognize unfamiliar objects in a 2AFC paradigm

267 (Fig. 1). Similar tasks have been developed for rats (Zoccolan et al., 2009), but mice have not been reported to 268 perform such a task. That might be related to the fact that even though mice and rats can achieve similar 269 performance levels, mice are slower to train (Jaramillo and Zador, 2014). Our unique training approach 270 involves minimal interactions with the animals since the training system is part of their housing. Within a few 271 weeks animals learn to discriminate objects and can show generalization across unseen objects poses 272 establishing that mice are capable of invariant object recognition (Fig. 1d). 
To identify how animals are able to extract object identity, we analyzed the activity of thousands of

274 neurons of all known visual cortical areas of the mouse. We found that the decoding performance varied

275 across the visual hierarchy. A set of lateral visual areas carried more linearly decodable information about the

276 object identity. Importantly, these areas retain the information about object identity even in difficult visual

277 conditions such as clutter and generalization across nuisance variability. Our results agree with the hypothesis

278 that object representations become untangled and more linearly separable as information progresses through

279 the visual hierarchy; this process might be beneficial as a simple readout mechanism may be employed in

280 order to use this information to drive behavior. It is important to note that a biologically plausible readout

281 mechanism would involve sampling only from a small set of neurons in order to extract object identity and

282 that was our motivation in restricting the access of the decoder to a small sample of neurons. Interestingly,

283 when computing the information per neuron we find that information also increased progressively across the

284 lateral hierarchy of V1-LM-LI as has been reported in electrophysiology studies in the rat (Tafazoli et al.,

285 2017) (Supp. Fig. 2a). However, we found that for larger populations area LI carries less information than

286 LM (Fig. 2a, b). This could be due to more redundant information between neurons in LI in the responses to

287 the specific set of objects used in this study. Therefore, analogous to primates in mice hierarchically organized

288 visual areas untangle pixel intensities into more linearly decodable object representations.

However, the associated changes in the geometry of the object manifold along the visual cortex

290 remain unknown for any species. To that end, we characterized how the geometry of the object manifolds

291 changed across the visual hierarchy, using the newly developed theory of linear separability of manifolds

292 (Chung et al., 2018; Cohen et al., 2020). We found that the two lateral visual areas LM and AL showed

293 increased classification capacity with manifolds that become smaller and have lower dimensionality (Fig. 4).

294 While the classification capacity and radius of object manifolds has not been previously quantified along the

295 visual processing hierarchy, our results on the dimensionality of the neural population agree with previous

296 work. Different methods have been used to quantify the dimensionality of the population responses which also

297 showed that it decreases along the visual hierarchy of monkeys (Brincat et al., 2018; Lehky et al., 2014).

298 However, critically the theory of the linear separability of manifolds differs from these previous methods as it

299 quantifies the geometrical properties of the object response manifolds which contribute to the ability to

300 perform linear decoding and thus enabled us to determine how the object manifold changes from primary to

301 higher visual areas in a way which allows for linear decoding of objects using smaller number of neurons.

302 The higher visual areas of the mouse (Glickfeld and Olsen, 2017; Wang and Burkhalter, 2007), have

303 distinct spatio-temporal selectivities (Andermann et al., 2011; Marshel et al., 2011) and project to different

304 targets (Wang et al., 2012). Based on these differences in their selectivities, projection and chemoarchitectonic

305 patterns, efforts have been made to separate areas into ventral and dorsal pathways analogous to those

306 described in primates (Smith et al., 2017; Wang et al., 2012, 2011; Wang and Burkhalter, 2013). Specifically,

307 areas such as LM, LI, P and POR areas are hypothesized to comprise the ventral stream whereas areas AL,

308 RL, AM and PM comprise the dorsal stream. In rats, lateral visual areas LM, LI and LL have been shown to

309 carry progressively more information about objects (Tafazoli et al., 2017; Vermaercke et al., 2015, 2014). 
310 However, the areas of the mouse that might be involved in extraction of object information are not known. We

311 found that higher visual areas AL, LM and LI had significantly more information about object identity than

312 V1, with area AL consistently outperforming all other areas which is not consistent with the current

313 assumption that AL is part of the dorsal pathway. In line with this, both areas AL and LM show faster

314 accumulation of information about object identity in noisy conditions (Supp. Fig. 6). Moreover, the

315 correlations in decoding confidence between areas AL and LM we find (Fig. 5c) could be the result of

316 recurrent processes that have been suggested to play a significant role during object recognition (Kar et al.,

317 2019; Tang et al., 2018, 2014). These object-selective dependencies do not share the same structure as have

318 been reported with more parametric stimuli (Smith et al., 2017), which could be due to objects having a

319 statistical structure closer to the preferences of these lateral visual areas.

Future experiments are required to determine how these different areas work together to extract

321 information about objects that might be used to guide behavior. In order to establish a more causal relationship

322 between visual areas and behavior, it will be important to combine behavioral performance with neural

323 activity manipulation. Neural networks models and the inception loop methodology will enable the

324 characterization of the specific features that drive neurons in these different visual areas (Bashivan et al.,

325 2019; Ponce et al., 2019; Walker et al., 2019).

In summary, we offer evidence that mice share similarities with rats and higher mammals in their

327 ability to recognize objects. We show that sequential visual processing leads to object manifolds with

328 decreased radius and dimension such that the manifolds are more linearly separable. Given the panoply of

329 tools available, the mouse has the potential to become a powerful model to dissect the circuit mechanisms of

330 object recognition. 


\section{Methods}

\section{Animal preparation and two photon imaging}

333 All procedures were approved by the Institutional Animal Care and Use Committee (IACUC) of Baylor 334 College of Medicine. We used 25 adult mice expressing GCaMP6s in excitatory neurons via either 335 SLC17a7-Cre, Dlx5-Cre, Ai75, Ai148, Ai162 or CamKII-tTA transgenic lines. Animals were initially 336 anesthetized with Isoflurane (2\%) and a $4 \sim \mathrm{mm}$ craniotomy was made over the right visual cortex as 337 previously described (Froudarakis et al., 2014). The animals were head-mounted above a cylindrical treadmill 338 and calcium imaging was performed using Chameleon Ti-Sapphire laser (Coherent) tuned to $920 \mathrm{~nm}$. We 339 recorded calcium traces by using either a large field of view mesoscope (Sofroniew et al., 2016) equipped 340 with a custom objective (0.6 NA, 21mm focal length) with a typical field of view of $\sim 2500 x 2000 \mu \mathrm{m}$, or a 341 two-photon resonant microscope (Thorlabs) equipped with a Nikon objective (1.1 NA, 25X) with a typical 342 field of view or $\sim 500 \times 500 \mu \mathrm{m}$. Laser power after the objective was kept below $\sim 60 \mathrm{~mW}$. We recorded data from 343 depths of $100-380 \mu \mathrm{m}$ below the cortical surface. Imaging was performed at approximately $5-12 \sim \mathrm{Hz}$ for all 344 scans. Imaging data were motion corrected, automatically segmented and deconvolved using the CNMF 345 algorithm (Pnevmatikakis et al., 2016); cells were further selected by a classifier trained to detect somata 346 based on the segmented cell masks.

\section{Behavioral training}

348 The mice are trained in a 2 alternative forced choice task in response to moving objects that are presented on a 349 small 7" monitor that is located in front of their home cage. The training procedure is illustrated in Figure 1. 350 Briefly, naive water unrestricted mice are placed in a modified cage that has three ports and a monitor on one 351 side of the box. The center port has a proximity sensor, and the two other ports on either side of the central 352 port are used to detect licks and are coupled to a computerized valve-controlled liquid delivery that can deliver 353 liquid volumes with $1 \mathrm{uL}$ resolution. The task is as follows: Mice initiate a trial by placing their snout in close 354 proximity to the central port for $\sim 200-500$ msec. A stimulus that can be one of two objects is presented on the 355 monitor that is $\sim 1.5$ ” in front of the animal. The animal has to report the identity of the object by licking one 356 of the side ports. Each port is allocated to the identity of the same object throughout the training. If the animal 357 licks the correct port, then a small water reward $\sim 5-12 \mu$ lis delivered almost immediately which the animals 358 consume. A new trial can be started thereafter. If the animal licks the wrong port, a short delay 4-10 seconds is 359 added and the screen turns black. A new trial can start after the delay. Animals have free access to food, and 360 the only water that they receive comes from their training. The training periods in which animals can initiate 361 tasks are restricted to 4-8 hours a day. At the start of the training animals are shown the same clip for each 362 object that contains the same set of transformations. Once animals reach performance levels, new clips with 363 unique transformations are added. At the end of their training they have seen between 10-20 unique 10s clips 364 of unique object transformations. For the generalization test, at the start of a new session a whole new set of 36510 clips are used for each object and the performance was compared to the session that preceded. 
Visual area identification

367 We generated retinotopic maps of all the visual areas using widefield imaging. The signals from GCamp6s

368 were captured using either a custom epifluorescence setup or two-photon imaging. For the epifluorescence,

369 brain was illuminated with a high power LED (Thorlabs) and the emitted signal was bandpass filtered at nm

370 and captured at a rate of $10 \mathrm{~Hz}$ with a CMOS camera (MV1-D1312-160-CL, PhotonFocus). For the

371 two-photon retinotopic mapping we sampled the activity from a $2.4 \times 2.4 \mathrm{~mm}$ area with large field of view two

372 photon microscope (Sofroniew et al., 2016) at a rate of $\sim 5 \mathrm{~Hz}$. We stimulated with upward and rightward

373 drifting white bars (speed: 9-18deg/sec, width: 10-20deg) on black background that had their size and speed

374 constant relative to the mouse perspective as previously described. Additionally, within the bar we had drifting

375 gratings with a direction opposite to the movement of the bar. Images from either the epifluorescent or the

376 two-photon setups were analyzed by a custom-written code in MATLAB to construct the 2D phase maps for

377 the two directions. We used the resulting retinotopic maps to identify the borders and delineate the visual areas

378 as previously described (Garrett et al., 2014; Wang and Burkhalter, 2007).

\section{Stimulus generation and visual stimulation}

380 In this study we used four synthesized three-dimensional objects that were rendered in Blender 381 (www.blender.org). Two of the objects were built to match the objects used in (Zoccolan et al., 2009) and the 382 other two were already existing models within Blender. We varied the following parameters of the objects: $\mathrm{X}$ 383 and Y location (Translation), magnification (Scale), tilt and axial rotation (Pose) and variation of either the 384 location or energy of 4 light sources (Light). The different object parameters were varied continuously over 385 time in order to generate a cohesive object motion. Objects were rendered either on a gray background, or on a 386 gaussian noise pattern with a fixed seed between objects. The long rendered movie was split into smaller 10 387 second clips. A short 3-5 second segment from 150-380 clips for each object were presented in a random 388 sequence to the left eye with a 25 " LCD monitor positioned $\sim 15 \mathrm{~cm}$ away from the animal. A small number of 389 clips were repeated multiple times in order to estimate the reliability of the neural responses.

\section{Decoding and discriminability}

391 We used a one-versus-all logistic regression classifier to estimate the decoding error between the neural 392 representations of 2-4 objects of 200-500 ms scenes. Each scene was represented as an N-dimensional vector 393 of neural activity for each response scene. In almost all of the cases we used a 10 fold cross-validation in 394 which the performance of the decoder was tested on $10 \%$ of the data that were held out during training. When 395 generalizing across the background noise in Figure 3, the decoder was trained on 90\% of the data with the 396 no-background objects and tested on $10 \%$ of the data with the background objects. For the generalization 397 across object parameters in Figure 3 we used a 4 fold cross-validation in which the decoder was trained on $39875 \%$ of the data , and tested on $25 \%$ with a unique parameter range. We converted the decoding error to 
399 discriminability, the mutual information (measured in bits) between the true class label $c$ and its estimate , by

400 computing

$401 M I(c, \widehat{c})=\sum_{i} \sum_{j} p_{i j} \log _{2} \frac{p_{i j}}{p_{i .} p_{j}}$

402 where $p_{i j}$ is the probability of observing true class $i$ and predicted class $j$ and $p_{i .}$ and $p_{. j}$ denote the respective 403 marginal probabilities.

\section{Classification capacity and geometry of manifolds}

405 An object manifold is defined by the neuronal population responses to an object under different conditions 406 (i.e. identity-preserving transformations). The ability of a downstream neuron to perform linear classification 407 of object manifolds depends on the number of objects, denoted $P$, and the number of neurons participating in 408 the representation, denoted $N$. Classification capacity denotes the critical ratio $\alpha_{c}=P / N_{c}$ where $N_{c}$ is the 409 population size required for a binary classification of $P$ manifolds to succeed with high probability (Chung et 410 al., 2018). This capacity can be interpreted as the amount of information about object identity coded per 411 neuron in the given population. Capacity $\alpha_{c}$ depends on the radius of each of the manifolds, denoted $R_{M}$, 412 representing the overall extent of variability (relative to the distance between manifolds), and their dimension, 413 denoted $D_{M}$, representing the number of directions in which this variability is spread. These geometric 414 measures are defined through the alignment of the hyperplane in the representation $N$-dimensional space that 415 separates positively labelled from negatively labelled manifolds. This hyperplane is uniquely determined by a 416 set of anchor points, one from each manifold, that lie exactly on the separating plane. As the classification 417 labels are randomly changed, the identity of the anchor points change, and these changes in addition to the 418 dependence of the hyperplane orientation on the particular position and orientation of the manifolds, give rise 419 to a statistical distribution of anchor points. Averaging the extent and directional spread of the anchor points 420 with this distribution determines the manifolds radii and dimensions, respectively. Knowledge of manifold 421 radius and dimension is sufficient to predict classification capacity using the relation $\alpha_{C}=\alpha_{\text {Balls }}\left(R_{M}, D_{M}\right)$ 422 where $\alpha_{\text {Balls }}$ is a closed-form expression describing capacity of $D$-dimensional balls of radius $R$ (Chung et 423 al., 2018).

424 The separability of manifolds depends not only on their geometries but also on their correlations. For manifold 425 classification with random binary labeling, clustering of the manifolds in the representational space, as 426 expected for real-world object representations, hinders their separability, and the theory of manifold 427 classification has been extended (Cohen et al., 2020) to take these correlations into account in evaluating $\alpha_{c}$.

428 Here we used the methods and code from (Cohen et al., 2020) to analyze the geometry of the object manifolds 429 (i.e. manifold radius and dimension) as well as estimate classification capacity of neuronal populations in the 430 different cortical areas. As those methods depend on the correlation structure of the objects, we analyzed 431 neural representations for data-sets of 4 objects (i.e. omitted data-sets where only 2 objects are available). At 432 each session of simultaneously recorded neurons we have sub-sampled from the available population 64 433 neurons; the subsequent analysis was repeated 10 times with different choices of neurons, and we report the 
434 average results across this procedure. Each object manifold is defined by neural responses to an object at 435 non-overlapping 500ms time windows, using the entire range of nuisance parameter space, as well as 436 responses with and without background noise. This analysis was performed at each visual area for sessions 437 where more than 64 neurons are available. The baseline to which classification capacity is compared is the 438 value expected by structure-less manifold which is $2 / M$, where $M$ is the number of samples. 


\section{References}

440

441

442

443

444

445

446

447

448

Andermann, M.L., Kerlin, A.M., Roumis, D.K., Glickfeld, L.L., Reid, R.C., 2011. Functional Specialization of Mouse Higher Visual Cortical Areas. Neuron 72, 1025-1039. https://doi.org/10.1016/j.neuron.2011.11.013

Bashivan, P., Kar, K., DiCarlo, J.J., 2019. Neural population control via deep image synthesis. Science 364, eaav9436. https://doi.org/10.1126/science.aav9436

Bevins, R.A., Besheer, J., 2006. Object recognition in rats and mice: a one-trial non-matching-to-sample learning task to study "recognition memory." Nature Protocols 1, 1306. https://doi.org/10.1038/nprot.2006.205

Blaser, R., Heyser, C., 2015. Spontaneous object recognition: a promising approach to the comparative study of memory. Front. Behav. Neurosci. 9. https://doi.org/10.3389/fnbeh.2015.00183

Brincat, S.L., Siegel, M., Nicolai, C. von, Miller, E.K., 2018. Gradual progression from sensory to task-related processing in cerebral cortex. PNAS 115, E7202-E7211. https://doi.org/10.1073/pnas.1717075115

Chung, S., Lee, D.D., Sompolinsky, H., 2018. Classification and Geometry of General Perceptual Manifolds. Physical Review X 8. https://doi.org/10.1103/PhysRevX.8.031003

Cohen, U., Chung, S., Lee, D.D., Sompolinsky, H., 2020. Separability and geometry of object manifolds in deep neural networks. Nature Communications 11, 746. https://doi.org/10.1038/s41467-020-14578-5

DiCarlo, J.J., Cox, D.D., 2007. Untangling invariant object recognition. Trends in Cognitive Sciences 11, 333-341. https://doi.org/10.1016/j.tics.2007.06.010

DiCarlo, J.J., Zoccolan, D., Rust, N.C., 2012. How Does the Brain Solve Visual Object Recognition? Neuron 73, 415-434. https://doi.org/10.1016/j.neuron.2012.01.010

Fahey, P.G., Muhammad, T., Smith, C., Froudarakis, E., Cobos, E., Fu, J., Walker, E.Y., Yatsenko, D., Sinz, F.H., Reimer, J., Tolias, A.S., 2019. A global map of orientation tuning in mouse visual cortex. bioRxiv 745323. https://doi.org/10.1101/745323

Fenno, L.E., Gunaydin, L.A., Deisseroth, K., 2015. Mapping Anatomy to Behavior in Thy1:18 ChR2-YFP Transgenic Mice Using Optogenetics. Cold Spring Harb Protoc 2015, pdb.prot075598. https://doi.org/10.1101/pdb.prot075598

Froudarakis, E., Berens, P., Ecker, A.S., Cotton, R.J., Sinz, F.H., Yatsenko, D., Saggau, P., Bethge, M., Tolias, A.S., 2014. Population code in mouse V1 facilitates readout of natural scenes through increased sparseness. Nat Neurosci 17, 851-857. https://doi.org/10.1038/nn.3707

Garrett, M.E., Nauhaus, I., Marshel, J.H., Callaway, E.M., 2014. Topography and Areal Organization of Mouse Visual Cortex. J. Neurosci. 34, 12587-12600. https://doi.org/10.1523/JNEUROSCI.1124-14.2014

Glickfeld, L.L., Olsen, S.R., 2017. Higher-Order Areas of the Mouse Visual Cortex. Annual Review of Vision Science 3, 251-273. https://doi.org/10.1146/annurev-vision-102016-061331

Han, X., Vermaercke, B., Bonin, V., 2018. Segregated encoding of spatiotemporal features in the mouse visual cortex. bioRxiv 441014. https://doi.org/10.1101/441014

Hénaff, O.J., Goris, R.L.T., Simoncelli, E.P., 2019. Perceptual straightening of natural videos. Nature Neuroscience 22, 984. https://doi.org/10.1038/s41593-019-0377-4

Hoy, J.L., Yavorska, I., Wehr, M., Niell, C.M., 2016. Vision drives accurate approach behavior during prey capture in laboratory mice. Current biology : CB 26, 3046. https://doi.org/10.1016/j.cub.2016.09.009

Hung, C.P., Kreiman, G., Poggio, T., DiCarlo, J.J., 2005. Fast Readout of Object Identity from Macaque Inferior Temporal Cortex. Science 310, 863-866. https://doi.org/10.1126/science.1117593

Jones, A.G., Ratterman, N.L., 2009. Mate choice and sexual selection: What have we learned since Darwin? PNAS 106, 10001-10008. https://doi.org/10.1073/pnas.0901129106

Kar, K., Kubilius, J., Schmidt, K., Issa, E.B., DiCarlo, J.J., 2019. Evidence that recurrent circuits are critical to the ventral stream's execution of core object recognition behavior. Nature Neuroscience 22, 974-983. https://doi.org/10.1038/s41593-019-0392-5

Leger, M., Quiedeville, A., Bouet, V., Haelewyn, B., Boulouard, M., Schumann-Bard, P., Freret, T., 2013. Object recognition test in mice. Nat. Protocols 8, 2531-2537. https://doi.org/10.1038/nprot.2013.155

Lehky, S.R., Kiani, R., Esteky, H., Tanaka, K., 2014. Dimensionality of object representations in monkey inferotemporal cortex. Neural Comput. 26, 2135-2162. https://doi.org/10.1162/NECO_a_00648 
Marshel, J.H., Garrett, M.E., Nauhaus, I., Callaway, E.M., 2011. Functional Specialization of Seven Mouse Visual Cortical Areas. Neuron 72, 1040-1054. https://doi.org/10.1016/j.neuron.2011.12.004

Murgas, K.A., Wilson, A.M., Michael, V., Glickfeld, L.L., 2020. Unique Spatial Integration in Mouse Primary Visual Cortex and Higher Visual Areas. J. Neurosci. 40, 1862-1873. https://doi.org/10.1523/JNEUROSCI.1997-19.2020

Newport, C., Wallis, G., Siebeck, U.E., 2018. Object recognition in fish: accurate discrimination across novel views of an unfamiliar object category (human faces). Animal Behaviour 145, 39-49. https://doi.org/10.1016/j.anbehav.2018.09.002

Orlov, T., Zohary, E., 2018. Object Representations in Human Visual Cortex Formed Through Temporal Integration of Dynamic Partial Shape Views. J. Neurosci. 38, 659-678. https://doi.org/10.1523/JNEUROSCI.1318-17.2017

Pnevmatikakis, E.A., Soudry, D., Gao, Y., Machado, T.A., Merel, J., Pfau, D., Reardon, T., Mu, Y., Lacefield, C., Yang, W., Ahrens, M., Bruno, R., Jessell, T.M., Peterka, D.S., Yuste, R., Paninski, L., 2016. Simultaneous Denoising, Deconvolution, and Demixing of Calcium Imaging Data. Neuron 89, 285-299. https://doi.org/10.1016/j.neuron.2015.11.037

Ponce, C.R., Xiao, W., Schade, P.F., Hartmann, T.S., Kreiman, G., Livingstone, M.S., 2019. Evolving Images for Visual Neurons Using a Deep Generative Network Reveals Coding Principles and Neuronal Preferences. Cell 177, 999-1009.e10. https://doi.org/10.1016/j.cell.2019.04.005

Rust, N.C., DiCarlo, J.J., 2010. Selectivity and Tolerance ("Invariance”) Both Increase as Visual Information Propagates from Cortical Area V4 to IT. J. Neurosci. 30, 12978-12995. https://doi.org/10.1523/JNEUROSCI.0179-10.2010

Smith, I.T., Townsend, L.B., Huh, R., Zhu, H., Smith, S.L., 2017. Stream-dependent development of higher visual cortical areas. Nat Neurosci 20, 200-208. https://doi.org/10.1038/nn.4469

Sofroniew, N.J., Flickinger, D., King, J., Svoboda, K., 2016. A large field of view two-photon mesoscope with subcellular resolution for in vivo imaging. eLife Sciences 5, e14472. https://doi.org/10.7554/eLife.14472

Soto, F.A., Wasserman, E.A., 2014. Mechanisms of object recognition: what we have learned from pigeons. Front Neural Circuits 8. https://doi.org/10.3389/fncir.2014.00122

Tafazoli, S., Safaai, H., Franceschi, G.D., Rosselli, F.B., Vanzella, W., Riggi, M., Buffolo, F., Panzeri, S., Zoccolan, D., 2017. Emergence of transformation-tolerant representations of visual objects in rat lateral extrastriate cortex. eLife 6, e22794. https://doi.org/10.7554/eLife.22794

Tang, H., Buia, C., Madhavan, R., Crone, N.E., Madsen, J.R., Anderson, W.S., Kreiman, G., 2014. Spatiotemporal Dynamics Underlying Object Completion in Human Ventral Visual Cortex. Neuron 83, 736-748. https://doi.org/10.1016/j.neuron.2014.06.017

Tang, H., Schrimpf, M., Lotter, W., Moerman, C., Paredes, A., Caro, J.O., Hardesty, W., Cox, D., Kreiman, G., 2018. Recurrent computations for visual pattern completion. PNAS 201719397. https://doi.org/10.1073/pnas.1719397115

Vermaercke, B., Gerich, F.J., Ytebrouck, E., Arckens, L., Op de Beeck, H.P., Van den Bergh, G., 2014. Functional specialization in rat occipital and temporal visual cortex. Journal of Neurophysiology 112, 1963-1983. https://doi.org/10.1152/jn.00737.2013

Vermaercke, B., Van den Bergh, G., Gerich, F., Op de Beeck, H., 2015. Neural discriminability in rat lateral extrastriate cortex and deep but not superficial primary visual cortex correlates with shape discriminability. Front. Neural Circuits 9. https://doi.org/10.3389/fncir.2015.00024

Walker, E.Y., Sinz, F.H., Cobos, E., Muhammad, T., Froudarakis, E., Fahey, P.G., Ecker, A.S., Reimer, J., Pitkow, X., Tolias, A.S., 2019. Inception loops discover what excites neurons most using deep predictive models. Nat. Neurosci. 22, 2060-2065. https://doi.org/10.1038/s41593-019-0517-x

Wang, Q., Burkhalter, A., 2013. Stream-Related Preferences of Inputs to the Superior Colliculus from Areas of Dorsal and Ventral Streams of Mouse Visual Cortex. J. Neurosci. 33, 1696-1705. https://doi.org/10.1523/JNEUROSCI.3067-12.2013

Wang, Q., Burkhalter, A., 2007. Area map of mouse visual cortex. J. Comp. Neurol 502, 339-357. https://doi.org/10.1002/cne.21286

Wang, Q., Gao, E., Burkhalter, A., 2011. Gateways of Ventral and Dorsal Streams in Mouse Visual Cortex. J. Neurosci. 31, 1905-1918. https://doi.org/10.1523/JNEUROSCI.3488-10.2011

Wang, Q., Sporns, O., Burkhalter, A., 2012. Network Analysis of Corticocortical Connections Reveals Ventral 
and Dorsal Processing Streams in Mouse Visual Cortex. J. Neurosci. 32, 4386-4399. https://doi.org/10.1523/JNEUROSCI.6063-11.2012

547 Werner, A., Stürzl, W., Zanker, J., 2016. Object Recognition in Flight: How Do Bees Distinguish between 3D 548 Shapes? PLOS ONE 11, e0147106. https://doi.org/10.1371/journal.pone.0147106

549 Zoccolan, D., Oertelt, N., DiCarlo, J.J., Cox, D.D., 2009. A rodent model for the study of invariant visual object recognition. PNAS 106, 8748-8753. https://doi.org/10.1073/pnas.0811583106 